\title{
LA TRADUCCIÓN ALEMÁN-ESPAÑOL DE TEXTOS MÉDICOS PERTENECIENTES AL ÁMBITO DE LA FISIOTERAPIA Y LA NEUROLOGÍA: ANÁLISIS TERMINOLÓGICO Y RECURSOS PARA EL TRADUCTOR
}

\author{
Maria del Carmen Balbuena Torezano \\ Universidad de Córdoba
}

\begin{abstract}
This paper focuses on the most important difficulties to translate into Spanish physiotherapeutic and neurological German texts. It will be analysed the terminology and those resources that the translator of medical texts has, in order to show possible solutions to the referred difficulties.
\end{abstract}

\section{INTRODUCCIÓN}

Una de las principales características del texto biosanitario es su alto grado de especialización, especialización que se pone de manifiesto en la existencia de una ingente variedad de escritos médicos, los cuales pueden clasificarse siguiendo distintos parámetros. Así, podemos encontrar, según la parte de la Medicina tratada, textos preclínicos, dentro de los cuales estarían los pertenecientes a la Anatomía, la Fisiología, la Microbiología, etc., y textos del ámbito de las especialidades clínicas, tales como aquellos concernientes a la Anestesiología, la Cardiología, la Cirugía, la Dermatología, la Endocrinología, la Ginecología, la Hematología, la Nefrología, la Neurocirugía, la Neurología, la Oftalmología, la Pediatría, la Psiquiatría, la Reumatología, la Traumatología y Ortopedia, la Urología, etc. Sin embargo, si tenemos en cuenta al destinatario de la publicación, encontraremos textos informativos, textos de divulgación, textos redactados para facultativos de medicina general, y por último, aquellos otros dirigidos a especialistas. Finalmente, según el tipo de publicación, cabe distinguir anuncios - de medicamentos, de prótesis, de aparatos ortopédicos, etc-, manuales, libros de bolsillo, monografias, fascículos, revistas de medicina - general o especializada-, prospectos farmacéuticos, suplementos especializados de diarios, certificados e informes médicos, etc.

Por ello, el traductor de textos biosanitarios debe adquirir una cualificación y una especialización determinada, en función del tipo de texto que haya de traducir y de la rama o especialidad a la que dicho texto pertenezca, y por supuesto, ha de tener un profundo conocimiento de la LO y la LM. Esto hace que el perfil idóneo para el ejercicio de la traducción biosanitaria alemán-español sea el de un médico con un conocimiento exhaustivo de ambas lenguas, o bien un traductor con profundos conocimientos de 
Medicina. No obstante, y como afirma Pilar Elena (2003: 143), existe un "hecho avalado por la realidad como es la práctica profesional de la traducción especializada, realizada por traductores no especialistas".

A continuación ofreceremos algunas consideraciones en torno a la traducción de dos tipologías de textos biosanitarios concretas: el texto médico perteneciente al ámbito de la Neurologia y aquel propio del ámbito de la Fisioterapia, y nos centraremos fundamentalmente en un breve análisis del léxico y en los principales recursos que tiene el traductor para solucionar problemas de tipo terminológico.

\section{Cómo TRADUCIR UN TEXTO MÉDICO: CONSIDERACIONES EN TORNO AL LÉXICO DEL TEXTO ORIGINAL (TO)}

\subsection{La comprensión del TO}

Toda traducción ha de iniciarse, sea cual fuere el TO, con la lectura detenida y la identificación de la temática general de dicho texto. Con respecto a la comprensión del TO biosanitario, Esperanza Alarcón afirma (Félix; Ortega 1998: 1027):

Si los textos médicos cubren un amplio espectro de variantes, también nos descubren aspectos particularmente relevantes. Las singulares características del traductor, a medio camino entre los conocimientos del profano y los del especialista le obligan a adoptar una mirada especialmente analítica, crítica y cauta con respecto a determinados términos y conceptos. En efecto, cualquier traductor profesional es capaz de comprender plenamente el texto que se dispone a traducir en el transcurso de la primera lectura. En cambio, cuando un texto es predominantemente especializado —en este caso, médico-, la fase de comprensión puede quedar incompleta.

Esta comprensión incompleta a la que alude Alarcón deriva, en parte, del empleo de tecnicismos y cultismos, así como de abreviaturas y acrónimos específicos, que sólo un especialista en Medicina —en el caso que hoy nos ocupa un neurólogo o un fisioterapeuta - o un traductor especializado puede comprender. Obsérvense las siguientes traducciones al castellano coloquial y al castellano biosanitario:

\begin{tabular}{|l|l|l|}
\hline \multicolumn{1}{|c|}{ LO LM (COLOQUIAL) } & \multicolumn{2}{|c|}{ LM (BIOSANITARIA) } \\
\hline $\begin{array}{l}\text { Der Patient sitzt } \\
\text { oder steht }\end{array}$ & $\begin{array}{l}\text { El paciente está sentado } \\
\text { ode pie }\end{array}$ & $\begin{array}{l}\text { El paciente está en } \\
\text { sedestación o en } \\
\text { bipedestación. }\end{array}$ \\
\hline $\begin{array}{l}\text { Der Schlaganfall } \\
\text { bezeichnet einen } \\
\text { neurologischen } \\
\text { Notfall }\end{array}$ & $\begin{array}{l}\text { El infarto cerebral } \\
\text { designa una crisis } \\
\text { neurológica }\end{array}$ & $\begin{array}{l}\text { El Accidente Vascular } \\
\text { Encefálico (AVE) designa } \\
\text { una crisis neurológica }\end{array}$ \\
\hline Hirntod & muerte cerebral & $\begin{array}{l}\text { encefalopatía hipóxica, } \\
\text { hipoxia cerebral }\end{array}$ \\
\hline
\end{tabular}


No obstante, existen herramientas y procedimientos mediante los cuales el traductor --especialmente el traductor novel- podrá ir creando su propia base de datos terminológica para que, de este modo, adquiera de forma progresiva los conocimientos necesarios para la traducción de este tipo de textos.

\subsection{Fase de documentación}

Una vez leído el TO e identificada su temática, se procede a la fase de documentación. Este es uno de los pasos clave para la labor del traductor, si éste desea lograr una traducción de calidad.

Qué duda cabe que también es preciso contar con diccionarios bilingües y monolingües que recojan el léxico perteneciente al campo semántico que aquí tratamos. No obstante, hemos de decir que son escasos los diccionarios bilingües alemán-español/español-alemán de términos médicos', y que no existe ningún diccionario de Neurología o de Fisioterapia que contengan los términos correspondientes en español y/o alemán. Sin embargo, el traductor podrá acudir a diccionarios terminológicos monolingües en ambas lenguas, para, de este modo, resolver con éxito la traducción de determinados términos.

¿Cómo puede entonces suplirse esta carencia de material de consulta para la búsqueda de términos desconocidos? Dado que la Neurología y la Fisioterapia son ciencias en continua evolución, y que despiertan el interés no sólo de la comunidad médica, sino también de muchos lectores ajenos al ámbito sanitario, es posible encontrar numerosos recursos en la red para acercarnos aún más al tema del TO que hemos de traducir.

Uno de los más útiles es el acceso gratuito a determinados diccionarios médicos on-line multilingües, como por ejemplo el traductor médico multilingüe que se encuentra en la página web http://www.tribunamedica.com/diccmedprinc.htm o bien el Glosario de términos médicos de la Unión Europea, que se encuentra en la página http://allserv.rug.ac.be/ rvdstich/eugloss/welcome.html. Las nuevas tecnologías también nos facilitan el acceso a determinados libros electrónicos. Así, existen páginas web como por ejemplo la de Free Books4Doctors ${ }^{2}$ en la que encontraremos hasta 650 libros electrónicos clasificados por especialidades y por títulos. Además, y según el título, las obras están clasificadas por idiomas, entre ellos, francés, inglés, alemán y español. A título de ejemplo, cabe mencionar la incursión de un diccionario sobre el SIDA (AIDS Taschenwörterbuch) en el que encontraremos todas las definiciones y abreviaturas relacionadas con tal enfermedad. En español, por ejemplo, existe también un manual sobre Neurología, destinado a una mejor formación de médicos no especialistas.

\footnotetext{
'Algunos de ellos son los siguientes: Tamayo Delgado, Juan, Medizinisches Wörterbuch Deutsch-Spanisch. Además, el autor tiene una página web (http://www.tamayo.de) en la que se encuentran algunos extractos del diccionario. Otros diccionarios son: Elservier's medical dictionary in five languages: English/American, French,Italian, Spanish and German (1964)..
}

${ }^{2}$ http://www.freebooks 4 doctors.com/fb/germ.htm. 
Otra posibilidad que internet nos ofrece es la búsqueda en distintos portales tanto españoles como alemanes de información sobre el tema central del TLO que hemos de traducir. Con ello también podremos aclarar conceptos y términos relativos a dicho tema.

\subsection{Consideraciones en torno al léxico del To.}

Ya centrados en el proceso de traducción, y más específicamente en el léxico, contemplaremos aquí cuestiones como el desconocimiento de determinados vocablos o la traducción de palabras inencontrables.

En lo concerniente al primer aspecto, el desconocimiento de la terminología, no sólo en alemán sino también en español, puede originar, a veces, días enteros de consulta. El léxico de esta tipología textual está nutrido en un alto porcentaje de helenismos y latinismos, lo cual puede facilitar la labor para traducir determinados vocablos. Un ejemplo de ello es el siguiente texto (Schlake, Roosen, 1996: 16)

Ausgehend von der Embryonalentwicklung, wird das Gehirn gewöhnlich in drei Abschnitte eingeteilt:

- das Rautenhirn (Rhombenzephalon)

- das Mittelhirn (Mesenzephalon)

- das Vorderhirn (Prosenzephalon)

Aus dem Rautenhirn differenzieren sich drei Hirnstrukturen:

- die Brücke (Pons)

- das verlängerte Mark (Medulla oblongata)

- das Kleinhim (Cerebellum)

Muchos de los términos que aparecen en el texto pertenecen al ámbito de la anatomía. En este caso, el traductor puede recurrir a atlas médicos, o a la nomenclatura anatómica actual, elaborada por el Comité Internacional de Nomenclatura Anatómica. No obstante, cabe señalar las siguientes observaciones:

1. La traducción literal del término Embryonalentwicklung es "desarrollo embrionario, desarrollo del embrión". No obstante, en anatomía, también se emplea el cultismo "morfogénesis".

2. Del mismo modo, la traducción literal del sustantivo Rautenhirn es "cerebro romboidal". Si consultamos atlas de anatomía, así como la mencionada nomenclatura, amén de diversas páginas web especializadas en la anatomía del cerebro y embriología humana, veremos que el término empleado es "cerebro posterior", dado el lugar en el que se encuentra.

3. Finalmente, das verlängerte Mark puede traducirse literalmente por "médula alargada, estirada", traducción desde luego demasiado ruda. En su lugar, en diccionarios generales alemán-español, como por ejemplo puede ser el Pons, al buscar el término alemán Mark encontramos la expresión verlängertes Rückmark, con el significado de "bulbo raquídeo". Para confirmar nuestras sospechas de traducción basta con buscar en la red en cualquier metabuscador 
esta última acepción española, y encontraremos que "bulbo raquídeo" y "médula oblongada" designan a una misma parte del cerebro. Por tanto, estamos en la dirección correcta.

De este modo, no resultará complicado encontrar la equivalencia terminológica en la lengua meta, siendo la traducción resultante la siguiente:

Partiendo de la morfogénesis, se suele dividir el encéfalo en tres partes:

- cerebro posterior (romboencéfalo)

- cerebro medio (mesencéfalo)

- cerebro anterior (prosencéfalo)

Saliendo del romboencéfalo se diferencian tres estructuras cerebrales:

- el puente (Pons)

- el bulbo raquídeo (médula oblongata)

- el cerebelo (Cerebellum)

No obstante, cabe señalar que en muchas ocasiones existe un término netamente alemán y otro de procedencia griega o latina. Por ello, es conveniente que el traductor conozca los principales sufijos, prefijos y raices de origen germánico, para, de este modo, poder relacionar ambos términos, el latino o el griego, y el alemán. Esto le facilitará mucho su búsqueda del término adecuado. Sirvan como ejemplos los siguientes pares de vocablos:

\begin{tabular}{|l|l|l|}
\hline $\begin{array}{c}\text { TÉRMINO DE } \\
\text { ORIGEN NO } \\
\text { ALEMÁN }\end{array}$ & \multicolumn{1}{|c|}{$\begin{array}{c}\text { TÉRMINO } \\
\text { ALEMÁN }\end{array}$} & \multicolumn{1}{|c|}{$\begin{array}{c}\text { TÉRMINO } \\
\text { ESPAÑOL }\end{array}$} \\
\hline $\begin{array}{l}\text { Apoplexie, } \\
\text { Apoplexia cerebro }\end{array}$ & $\begin{array}{l}\text { Hirnanfall, Hirninfarkt, } \\
\text { Schlaganfall }\end{array}$ & $\begin{array}{l}\text { Accidente Vascular } \\
\text { Encefálico, Accidente } \\
\text { cerebro-vascular, } \\
\text { infarto cerebral }\end{array}$ \\
\hline Enzephalitis & Gehirnentzündung & encefalitis \\
\hline Meningitis & Hirnhautentzündung & meningitis \\
\hline Cephalgie, Kephalgie & Kopfschmerzen & Cefalea \\
\hline Commotio cerebro & Gehirnerschütterung & conmoción cerebral \\
\hline
\end{tabular}

Otro de los rasgos especificos de la terminología médica en general, y de la Neurología y la Fisioterapia en particular, es el empleo de abreviaturas y acrónimos. Así, tenemos, por ejemplo: 


\begin{tabular}{|c|c|c|}
\hline $\begin{array}{c}\text { ABREVIATURA/ACRÓNI } \\
\text { MO EN LO }\end{array}$ & $\begin{array}{l}\text { TÉRMINO } \\
\text { EN LO }\end{array}$ & $\begin{array}{c}\text { TÉRMINO EN } \\
\text { LM }\end{array}$ \\
\hline AST & Ausgangsstellung & posición inicial \\
\hline BWS & Brustwirbelsäule & $\begin{array}{l}\text { columna vertebral } \\
\text { dorsal }\end{array}$ \\
\hline CT & $\begin{array}{l}\text { Computer- } \\
\text { tomographie }\end{array}$ & $\begin{array}{l}\text { tomografia } \\
\text { computarizada }\end{array}$ \\
\hline DMS & $\begin{array}{l}\text { Durchblutung, } \\
\text { Motorik und } \\
\text { Sensibilität }\end{array}$ & $\begin{array}{l}\text { riego sanguíneo, } \\
\text { motricidad y } \\
\text { sensibilidad }\end{array}$ \\
\hline EEG & $\begin{array}{l}\text { Elektroenzephalogr } \\
\text { amm }\end{array}$ & Electroencefalograma \\
\hline HMSN I & $\begin{array}{l}\text { Hereditäre } \\
\text { motorische und } \\
\text { sensorische } \\
\text { Neuropathie Typ I }\end{array}$ & $\begin{array}{l}\text { Neuropatía senso- } \\
\text { motora hereditaria, } \\
\text { tipo I. }\end{array}$ \\
\hline HWS & Halswirbelsäule & $\begin{array}{l}\text { columna vertebral } \\
\text { cervical }\end{array}$ \\
\hline LWS & Lendenwirbelsäule & $\begin{array}{l}\text { columna vertebral } \\
\text { lumbar }\end{array}$ \\
\hline NMR/KST & $\begin{array}{l}\text { Magnet-Resonanz- } \\
\text { tomographie/ } \\
\text { Kernspin- } \\
\text { tomographie }\end{array}$ & $\begin{array}{l}\text { Resonancia } \\
\text { Magnética Nuclear } \\
(\mathrm{RMN})\end{array}$ \\
\hline PIR & $\begin{array}{l}\text { Postisometrische } \\
\text { Relaxation }\end{array}$ & $\begin{array}{l}\text { relajación } \\
\text { postisométrica }\end{array}$ \\
\hline TRP & Triggerpunkte & Puntos gatillo \\
\hline
\end{tabular}

A continuación ofrecemos dos ejemplos de textos donde es frecuente el uso de acrónimos y abreviaturas. El primero de ellos (Ehmer 2003: 167) pertenece al ámbito de la Fisioterapia; el segundo, a la Neurología (Schlake, Roosen 1996: 21):

Texto 1:

$[\ldots] \ldots$ 


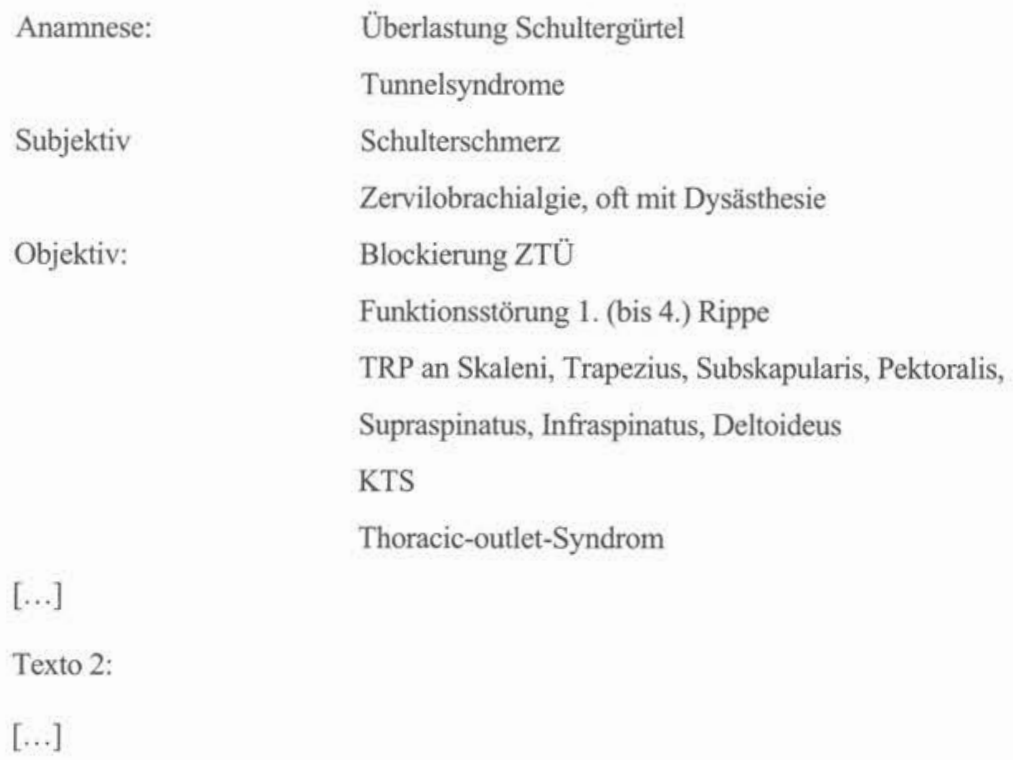

Im Gegensatz hierzu ist der Vorderlappen (HVL) Bildungsort einer Vielzahl von Hormonen; die wichtigsten sind: das Wachstumshormeon (STH), das die Schilddrüse stimulierende thyreotrophe Hormon (TSH), das auf die Nebenniere wirkende adrenocorticiptrophe Hormon (ACTH), die auf Geschlechtsorgane einwirkenden Gonadortropine sowie Prolaktin, welches die Milchsekretion in den Brustdrüsen stimuliert.

Por otra parte, es frecuente encontrar numerosos epónimos, que responden al nombre propio del científico que descubrió una determinada enfermedad, que practicó un determinado procedimiento quirúrgico, o que inventó algún mecanismo, aparato o maquinaria para el diagnóstico y el tratamiento de una dolencia determinada. Observemos las siguientes expresiones:

\begin{tabular}{|l|l|}
\hline \multicolumn{1}{|c|}{ TÉRMINO EN LO } & \multicolumn{1}{c|}{ TÉRMINO EN LM } \\
\hline Junghanssche Bewegungssegment & Segmento de movimiento de Junghans \\
\hline Laséguesche Zeichen & Signo/Prueba de Lasègue \\
\hline Klappsches Kriechen & (Ejercicios de) gateo de Klapp \\
\hline Schmorlsche Knötchen & Nódulos de Schmorl \\
\hline Alzeihmer Erkrankung & enfermedad de Alzheimer \\
\hline Guillain-Barré-Syndrom & síndrome de Guillain-Barré \\
\hline
\end{tabular}


Del mismo modo, los descubrimientos de nuevas técnicas y tratamientos, fármacos y demás, originan la aparición de neologismos y de anglicismos. Como ejemplo, tenemos los siguientes pares de vocablos:

\begin{tabular}{|l|l|}
\hline \multicolumn{1}{|c|}{ TÉRMINO EN LO } & \multicolumn{1}{c|}{ TÉRMINO EN LM } \\
\hline ADL = Activity of daily live & actividad de la vida diaria \\
\hline Alltagstraining & entrenamiento diario \\
\hline Haltungstraining & entrenamiento postural \\
\hline Impingement-Syndrom & sindrome de pinzamiento \\
\hline Joint play & juego articular \\
\hline
\end{tabular}

No hemos de olvidar tampoco que en un lenguaje tan específico como es el lenguaje biosanitario y por consiguiente, el de los textos físioterapéuticos y neurológicos, también encontramos falsos amigos, algunos de los cuales presentamos aquí:

\begin{tabular}{|c|c|c|}
\hline TÉRMINO EN LO & $\begin{array}{c}\text { TÉRMINO } \\
\text { INCORRECTO EN LA } \\
\text { LM }\end{array}$ & $\begin{array}{c}\text { TÉRMINO } \\
\text { CORRECTO EN LA } \\
\text { LM }\end{array}$ \\
\hline Balance & Balance & Equilibrio \\
\hline Ballon & Balón & $\begin{array}{l}\begin{array}{l}\text { Globo p. } \\
\text { Ballonkatheter } \\
\text { con globo) }\end{array} \\
\text { (catéter }\end{array}$ \\
\hline Band & Banda & Ligamento \\
\hline Blutdruck & Presión sanguínea & Presión o tensión arterial \\
\hline Dekubitus & Decúbito & $\begin{array}{l}\text { En alemán designa las } \\
\text { escaras por decúbito o } \\
\text { úlceras por decúbito }\end{array}$ \\
\hline Extension & Extensión (Streckung) & Tracción \\
\hline Extensiontisch & Mesa de extensión & Mesa de tracción \\
\hline Hypertonie & $\begin{array}{l}\text { Hipertonía (Hypertonus, } \\
\text { Muskelhypertonie) }\end{array}$ & Hipertensión arterial \\
\hline Hypotonie & $\begin{array}{l}\text { Hipotonía (Hypotonus, } \\
\text { Muskelhypotonie) }\end{array}$ & Hipotensión arterial \\
\hline Kollumfraktur & Fractura de columna & $\begin{array}{l}\text { Fractura del cuello de un } \\
\text { hueso largo }\end{array}$ \\
\hline Krankengymnastik & Gimnasia de los enfermos & Fisioterapia, cinesiterapia \\
\hline Krankengymnast & Gimnasta & Fisioterapeuta \\
\hline Kur & Cura & Tratamiento \\
\hline Operativ & Operativo & Quirúrgico, operatorio \\
\hline
\end{tabular}




\begin{tabular}{|l|l|l|}
\hline Orthese & Ortesis & Ortosis \\
\hline Quadrizepsreflex & Reflejo del cuádriceps & Reflejo rotuliano \\
\hline Reposition & Reposición & $\begin{array}{l}\text { La maniobra de } \\
\text { recolocación de un hueso } \\
\text { en caso de fractura o } \\
\text { luxación no se llama en } \\
\text { castellano reposición, } \\
\text { sino reducción }\end{array}$ \\
\hline Sektion & Sección & $\begin{array}{l}\text { Necropsia, disección } \\
\text { ecografiagrafia, }\end{array}$ \\
\hline Sonographie & Sonografía \\
\hline
\end{tabular}

En ocasiones, el traductor podrá encontrar términos que no tienen una fácil traducción, en parte porque no se denominan de igual modo en español, y en parte, porque no existen en la realidad española. Tomemos como ejemplo la traducción de la siguiente oración: "Entlastungsstellungen können im Stufenbett oder im Schlingentisch erzielt werden". Si recurrimos a la fragmentación de cada uno de los elementos que forman la composición sustantiva, obtendremos una traducción literal del término Schlingentisch que tal vez pueda ayudarnos a entender el concepto. En lo concerniente al primer término de la composición, Schlingen significa "cabestrillo", que unido a -tisch daría como resultado la traducción literal "mesa de cabestrillos". Para verificar esa intuición que tenemos sobre el concepto, y afianzar y comprender mejor de qué se trata, podemos recurrir a la red, que, si bien es cierto que no siempre ofrece soluciones, sí puede aportarnos elementos y herramientas auxiliares que nos ayuden a realizar la traducción. Si empleamos cualquier metabuscador, encontraremos numerosos enlaces en los que aparece este término.

No obstante, y como apuntábamos al principio, en caso de duda, amén de los diccionarios y recursos electrónicos, un especialista podrá indicarnos, previa "descripción" o "traducción literal" del término, qué expresión en LM es la más adecuada. La traducción al español es, finalmente, "mesa de cabestrillos". Algo parecido ocurre con Stufenbett. Si fragmentamos el vocablo, obtenemos los sustantivos Stufe (escalón, peldaño) y Bett (cama). La traducción literal "cama de peldaños, cama de escalones" no es adecuada. Se trata, en efecto, de camas articuladas que pueden posicionarse en distintos niveles. Por ello, la traducción correcta sería "cama articulada". Por tanto, la traducción de toda la oración sería: "Las posiciones de descarga pueden lograrse en una cama articulada o en una mesa de cabestrillos".

Un ejemplo aplicado a la Neurología, es el siguiente diagrama, que hace referencia a la aparición de crisis epilépticas: 


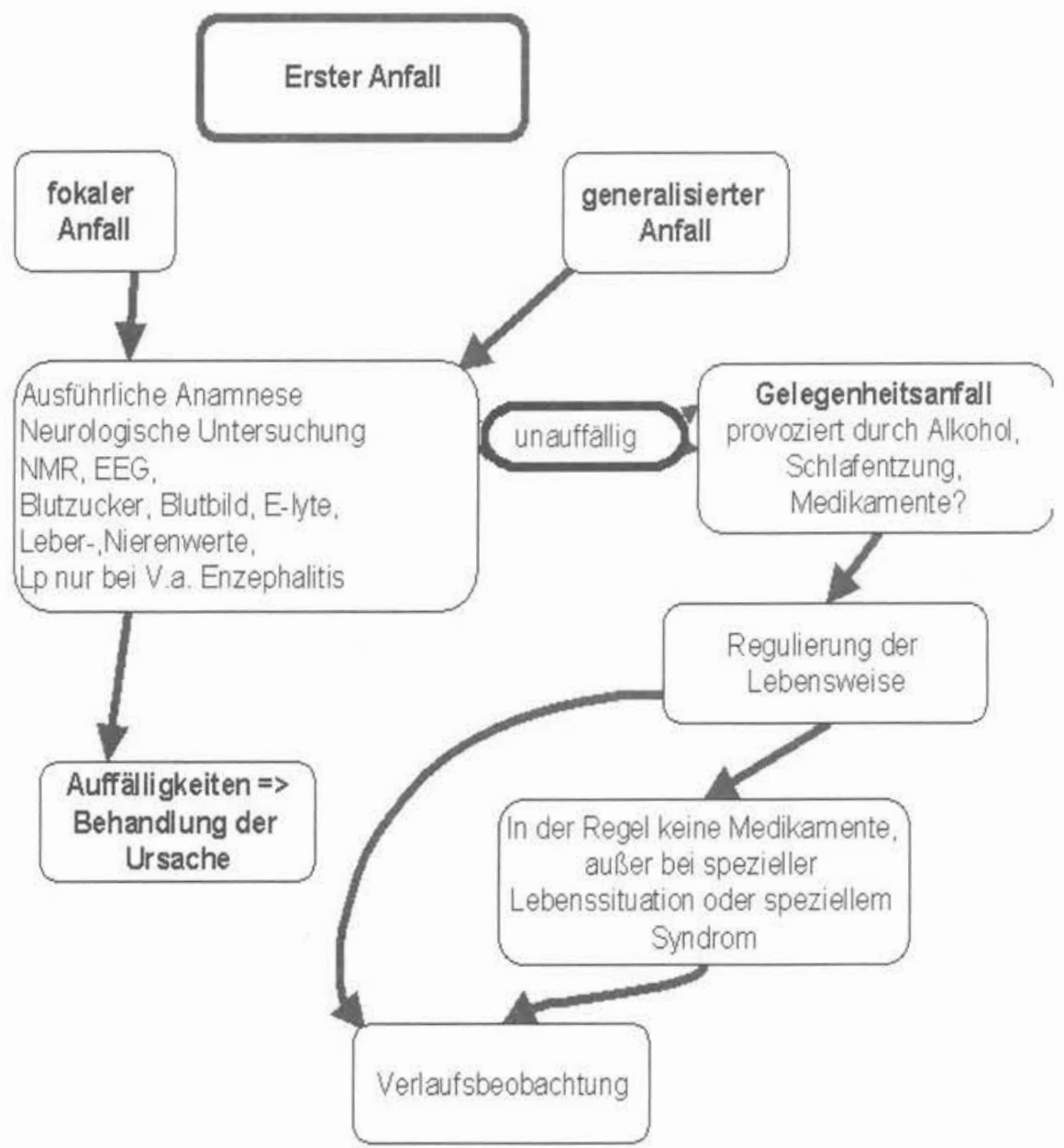

Las principales observaciones en cuanto a la traducción de los términos son las siguientes:

- En primer lugar, la traducción literal del término fokal, esto es, "focal", resulta demasiado forzada en castellano, por lo que el sentido médico propiamente dicho es "parcial".

- En segundo lugar, hemos de tener en cuenta la traducción de abreviaturas tales como NMR, tomada del inglés Nuclear Magnetic Resonance, y equivalente en castellano a "Resonancia Magnética Nuclear", que de forma abreviada se escribe RMN; o bien EEG, Elektroenzephalogramm, que equivale al término español "Electroencefalograma" y cuya abreviatura coincide con la alemana (EEG). Algo más dificiles de traducir son las abreviaturas Lp (Lumbalpunktion), "punción lumbar" y V. a. (Verdacht auf), "sospecha de". 
- Finalmente, la traducción de Auffälligkeiten, tomando el significado del adjetivo auffällig (novedoso, algo que llama la atención)

El texto resultante en LM sería, entonces, el siguiente:

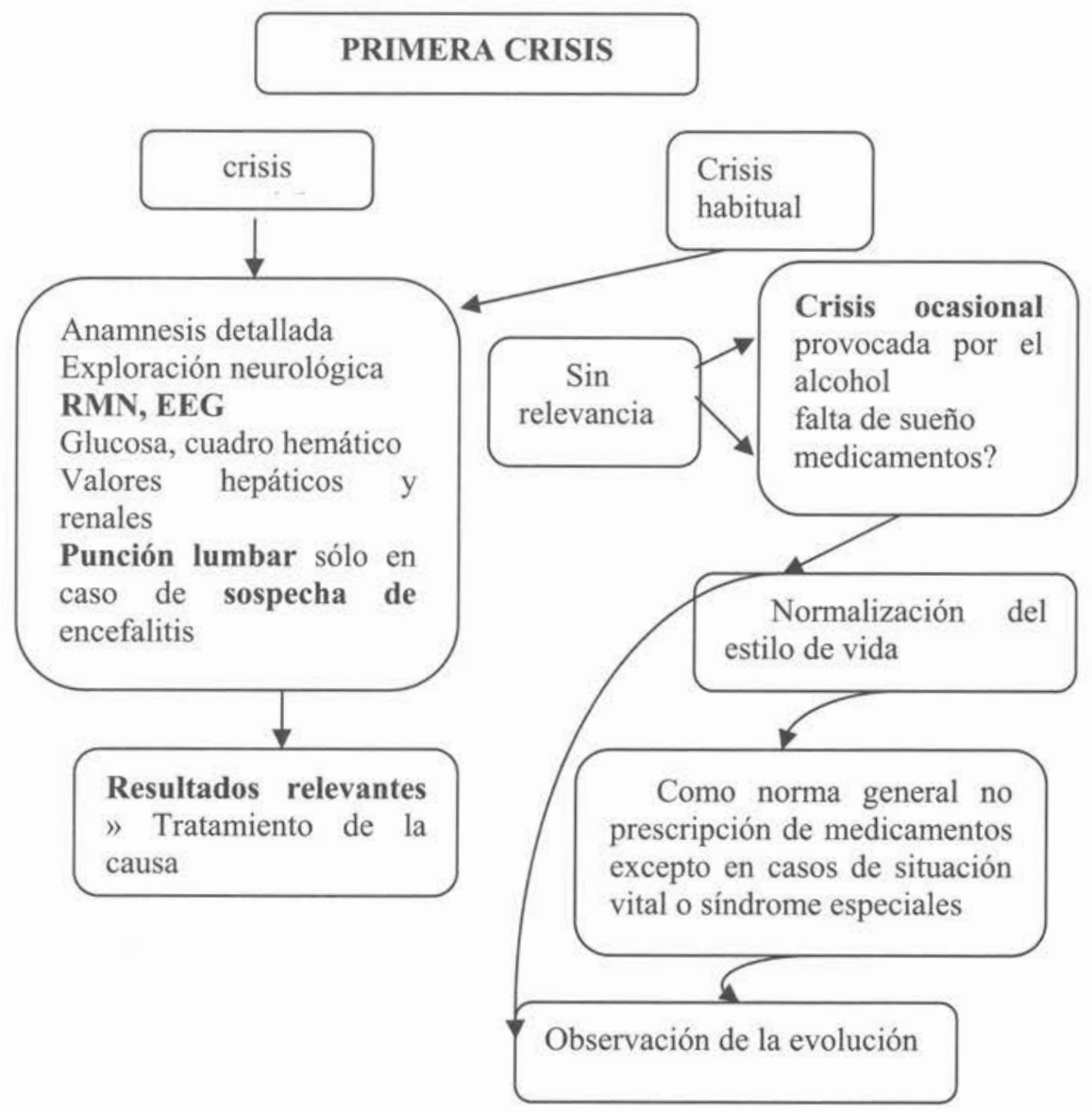

\section{CONCLUSIONES}

El recorrido que hemos efectuado por algunos de los aspectos léxicos y terminológicos en lo que a la traducción de textos especializados de Neurología y Fisioterapia se refiere, nos conduce a las siguientes conclusiones:

1. Las propias características del lenguaje propio de la Medicina implican la necesidad de especialización del traductor en función de la especialidad sobre la cual verse el texto médico a traducir. 
2. Existen recursos mediante los cuales podemos avanzar en la traducción del texto, si bien hemos de tener en cuenta las características especiales del léxico propio de esta tipología textual.

3. La traducción de este tipo de textos supone un amplio conocimiento de la lengua alemana, y más concretamente, del alemán especializado, de la medizinische Fachssprache, que implica una formación lingüística muy concreta y determinada. Esto mismo es necesario en la lengua meta, esto es, es necesario el conocimiento de terminología, expresiones y la fraseología propias del lenguaje médico en español.

4. No obstante, el aprendizaje mediante una formación dirigida y específica, que tenga muy en cuenta los aspectos léxicos, semánticos y terminológicos, puede contribuir a la especialización del futuro traductor.

\section{REFERENCIAS BIBLIOGRÁFICAS}

EHMER, B., Orthopädie und Traumatologie für Physiotherapeuten, Stuttgart, Hippokrates Verlag, 2003.

EHMER, B., Fisioterapia en Ortopedia y Traumatología, Traducción de Carmen Balbuena Torezano, Pedro Calle Fuentes, Miguel Muñoz-Cruzado y Barba y Felipe Ruiz Ayuso, Madrid, McGraw-Hill Interamericana, 2005.

FÉLIX FERNÁNDEZ, L. y E. ORTEGA ARJONILLA (coord.), Traducción en el ámbito biosanitario, Granada, Comares, 1998.

FISCHBACH, H. (ed.), Translation and Medicine, Amsterdam, John Benjamins, 1998.

NAVARRO, F. A., Traducción y lenguaje en medicina, Barcelona, Fundación Dr. Antonio Esteve, 1997.

SCHLAKE, H.-P.; KL., ROOSEN, Der Hirntod als der Tod des Menschen, Würzburg, Deutsche Stiftung Organtransplantation, 1996. 